\title{
Double-sided Polishing Photonic Crystal Fiber Biosensor Based on Surface Plasmon Resonance
}

\section{Zhiwen Zhang}

Harbin University of Science and Technology

tao shen ( $\nabla$ taoshenchina@163.com )

Harbin University of Science and Technology https://orcid.org/0000-0001-6357-6048

Chi Liu

Harbin University of Science and Technology

Xin Liu

Harbin University of Science and Technology

Yue Feng

Harbin University of Science and Technology

\section{Shaofeng Wang}

Harbin University of Science and Technology

\section{Han Wei}

Fujian Normal University

\section{Research Article}

Keywords: Fiber optic biosensor, Double-sided polishing photonic crystal fiber, Surface plasmon resonance, Cancer detection sensor

Posted Date: November 10th, 2021

DOI: https://doi.org/10.21203/rs.3.rs-949611/v1

License: (c) (1) This work is licensed under a Creative Commons Attribution 4.0 International License.

Read Full License 
Double-sided polishing photonic crystal fiber biosensor based on surface plasmon resonance

\title{
Zhiwen Zhang ${ }^{1}$, Chi Liu ${ }^{1,2}$, Xin Liu'1,3, Yue Feng ${ }^{1,2}$, Shaofeng Wang ${ }^{1}$, Tao Shen ${ }^{1,2,3,4,5 *}$, Han Wei ${ }^{4, \#}$
}

\author{
${ }^{1}$ Heilongjiang Province Key Laboratory of Laser Spectroscopy Technology and Application, Harbin \\ University of Science and Technology, Harbin 150080, China;
}

${ }^{2}$ Key Laboratory of Engineering Dielectrics and Its Application, Ministry of Education, Harbin University of Science and Technology, Harbin, 150080, China.

${ }^{3}$ Heilongjiang Provincial Key Laboratory of Quantum Control, Harbin University of Science and Technology, Harbin 150080, China

${ }^{4}$ Key Laboratory of Intelligent Optical Sensing and Manipulation (Nanjing University), Ministry of Education. China;

${ }^{5}$ Digit Fujian Internet-of-Things Laboratory of Environmental Monitoring, Fujian Normal University, Fuzhou 350117, China;

*Corresponding author: taoshenchina@163.com (Shen Tao).

\begin{abstract}
In this paper, we propose a high-sensitivity double-sided polishing photonic crystal fiber biosensor based on surface plasmon resonance. The performance of the sensor was analyzed by finite element method, including the influence of different pore sizes and position, polishing depth, pore spacing, metal thickness, refractive index(RI), and manufacturing error $(5 \%)$ on the sensing performance. We also analyze the performance of the modified sensor in early cancer detection applications. Our use of a double-sided polishing structure and coating with a gold layer not only increases the contact area between the analyte and gold layer but also provides a large enough space for the analysis channel, which is conducive to the actual manufacture and use. The sensor has a detection range of 1.35-1.39, the maximum wavelength sensitivity of $21500 \mathrm{~nm} / \mathrm{RIU}$, the average wavelength sensitivity is $9550 \mathrm{~nm} / \mathrm{RIU}$, the amplitude sensitivity of $-1057.1756 \mathrm{RIU}^{-1}$, the maximum resolution is $4.68 \times 10^{-6}, \mathrm{R}^{2}$ of 0.98265 , figure of merit is 250 , and the maximum resolution of $4.68 \times 10^{-6}$, which can be used in the cancer detection and other biological areas.
\end{abstract}

Keywords: Fiber optic biosensor, Double-sided polishing photonic crystal fiber, Surface plasmon resonance, Cancer detection sensor.

\section{Introduction}

At present, surface plasmon resonance (SPR) sensors are used in a variety of fields, such as environmental gas detection, biochemical medical analysis, and food safety [1]. The sensor has the characteristics of real-time, high sensitivity, and remote control [2,3]. Kretschmann experimented with 
SPRS in 1968, but it was expensive, bulky, and unreliable [4,5]. These shortcomings make PRISM SPR unable to distributed sensing and mass production [6,7].

To solve this problem, a photonic crystal fiber sensor based on surface plasmon resonance is developed by combining photonic crystal fiber with surface plasmon resonance (PCF-SPR) [8-10]. This kind of sensor has the advantages of flexible structure, anti-electromagnetic interference, high integration, and high mechanical stability [11,12]. In 2015, Sharma et al. proposed a photonic crystal fiber sensor for cancer detection [13]. In 2018, Liu et al. designed a D-shaped photonic crystal fiber biosensor with a wavelength sensitivity of $3340 \mathrm{~nm} / \mathrm{RIU}$ [14]. In 2020, Yasli developed a photonic crystal fiber biosensor to detect six types of cancer single cells [15]. In the field of biosensors, since the refractive index of blood cells, cervical cells, skin basal cells, adrenaline, and breast cells are all in the range of 1.35-1.39 [16], it is meaningful to propose a biosensor with a detection range of 1.35-1.39. However, to obtain higher sensitivity, people have studied the D-shape PCF-SPR to carry out deeper polishing to increase the contact area with metal, which will lead to a sharp increase in transmission loss, which is not conducive to detection [17]. Some other PCF-SPR sensors are also difficult to be used in practical applications due to their impractical analyte channels.

This paper presents a PCF-SPR sensor with double-sided film polishing. It has both realizable analyte channels and low confinement loss. The maximum wavelength sensitivity of the sensor proposed in this paper is $21500 \mathrm{~nm} / \mathrm{RIU}$, the average wavelength sensitivity is $9550 \mathrm{~nm} / \mathrm{RIU}$, the amplitude sensitivity is $-1057.1756 \mathrm{RIU}^{-1}$, and the maximum resolution is $4.68 \times 10^{-6}$, which can realize the monitoring between RI 1.35-1.39, and can be applied in the biomedical field in practice.

\section{The structure of the PCF and basic theory}

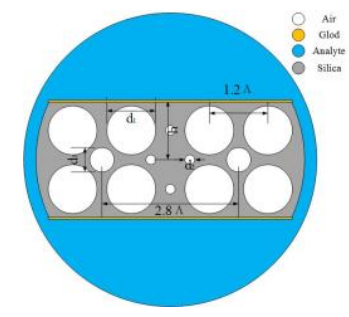

Fig. $12 \mathrm{D}$ cross-sectional view of the PCF-SPR sensor.

Fig. 1 is a 2D cross-section of the PCF-SPR sensor model proposed in this paper. The diameter of air holes $\mathrm{d}_{1}, \mathrm{~d}_{2}$ and $\mathrm{d}_{3}$ are $2.0 \mathrm{um}, 0.4 \mathrm{um}$, and $1.0 \mathrm{um}$ respectively. We set $\Lambda$ to $2.0 \mathrm{um}$, spacing $\mathrm{d}_{1}$ to $1.2 \Lambda$, spacing $\mathrm{d}_{2}$ to $1.6 \Lambda$ transverse, spacing $2.4 \Lambda$ longitudinal, and spacing $\mathrm{d}_{3}$ to $2.8 \Lambda$. The large air hole is arranged in a simple quadrilateral shape, which helps to reduce the production difficulty. The air hole $\mathrm{d}_{2}$ is arranged in a rhombic shape, which can increase the coupling strength of y polarization. The air hole $d_{2}$ effectively limits the mode field position of the core mode. The $h$ is the height from the PCF center to the casting mold position, and the gold layer thickness $t$ is set to $40 \mathrm{~nm}$. The gold layer can be coated by magnetron sputtering [18] or chemical vapor deposition [19]. The polishing mold can be polished with the edge arc glass base method and the polishing accuracy is $0.01 \mathrm{um}$ [20].

The dielectric constant of silica is calculated by Sellmeier equation [21]:

$$
n^{2}(\lambda)=1+\frac{A_{1} \lambda^{2}}{\lambda^{2}-B_{1}}+\frac{A_{2} \lambda^{2}}{\lambda^{2}-B_{2}}+\frac{A_{3} \lambda^{2}}{\lambda^{2}-B_{3}}
$$

Where $A_{1}, A_{2}, A_{3}, B_{1}, B_{2}$, and $B_{3}$ are $0.696166300,0.407942600,0.897479400,4.67914826 \times 10^{-3}$ $\mu \mathrm{m}^{2}, 1.35120631 \times 10^{-2} \mu \mathrm{m}^{2}$, and $97.9340025 \mu \mathrm{m}^{2}$, respectively. There, $\lambda$ is the incident light wavelength in a vacuum. 
The wavelength dependence on the dielectric constant of the gold layer is calculated using the Drude-Lorentz model [22]:

$$
\varepsilon_{A u}=\varepsilon_{\infty}-\frac{\omega_{D}^{2}}{\omega\left(\omega+j \gamma_{D}\right)}-\frac{\Delta \varepsilon \Omega_{L}^{2}}{\left(\omega^{2}-\Omega^{2}\right)+j \Gamma_{L} \omega}
$$

Where $\gamma_{D}$ and $\omega_{D}$ are the damping and plasma frequency, respectively. Here $\Delta \varepsilon$ is the permittivity at a high frequency, having a value of 5.9673, and $\varepsilon_{\mathrm{Au}}$ represents the permittivity of Au. Additionally, other parameters in the model have the numerical value of $\omega_{\mathrm{D}} / 2 \pi=2113.6 \mathrm{THz}, \gamma_{\mathrm{D}} / 2 \pi=15.92 \mathrm{THz}$, and $\Delta \varepsilon=1.09$. Note that, the spectral width and the oscillator strength is $\Gamma_{\mathrm{L}} / 2 \pi=104.86 \mathrm{THz}$ and $\Omega_{\mathrm{L}} / 2 \pi=650.07$ THz.

The confinement loss is used to analyze the characteristics of the sensor and can be evaluated by the following equation [23]:

$$
\operatorname{Loss}(\mathrm{dB} / \mathrm{cm}) \approx 8.86 \times \frac{2 \pi}{\lambda} \times \operatorname{Im}\left(n_{\text {eff }}\right) \times 10^{4}
$$

$\operatorname{Im}\left(\mathrm{n}_{\text {eff }}\right)$ is the virtual part of the effective index of the fundamental mode, which can be obtained by COMSOL software, $\lambda$ is the working wavelength.

In this paper, the sensitivity of the sensor is calculated by wavelength and amplitude interrogation methods. The formula of wavelength sensitivity is [24]:

$$
S_{\omega}(n m / R I U)=\frac{\Delta \lambda_{\text {peak }}}{\Delta n_{a}}
$$

In the formula, $\Delta \lambda_{\text {peak }}$ is the peak wavelength shift, and the $n_{a}$ is the change of the refractive index (RI) of the analyte.

The amplitude sensitivity formula is [25]:

$$
S_{A}\left(R I U^{-1}\right)=-\frac{1}{\alpha\left(\lambda, n_{a}\right)} \frac{\delta \alpha\left(\lambda, n_{a}\right)}{\delta n_{a}}
$$

In the formula, $\alpha\left(\lambda, n_{a}\right)$ is the confinement loss at the $n_{a}$ analyte RI, and $\delta \alpha\left(\lambda, n_{a}\right)$ is the difference of the confinement loss generated by two adjacent RI of the analyte.

The resolution equation is [26]:

$$
R(R I U)=\frac{\Delta n_{a} \times \Delta \lambda_{\min }}{\Delta \lambda_{\text {peak }}}
$$

Among them, $\Delta \lambda_{\min }$ is the resolution of the spectrometer, which is usually set at $0.1 \mathrm{~nm}$.

Figure of merit(FOM) is an important parameter for measuring sensor performance by combining sensitivity and full-width-half-maximum(FWHM), which is defined as [27] :

$$
F O M=\frac{S_{\omega}}{F W H M}
$$

The PCF is usually connected to a single-mode fiber (SMF). At this point, a broadband light source is used to transmit the light into the PCF via the SMF. The sensing process is based on the coupling between the surface plasmon polariton(SPP) mode and the core mode. When the refractive index of the two effective modes is the same, a sharp loss peak will result in the coupling. When the refractive index of the analyte changes, the resonance wavelength changes accordingly. The PCF can sense this change and output it to a high-resolution spectrograph for analysis [28]. 


\section{Simulation results and analysis}

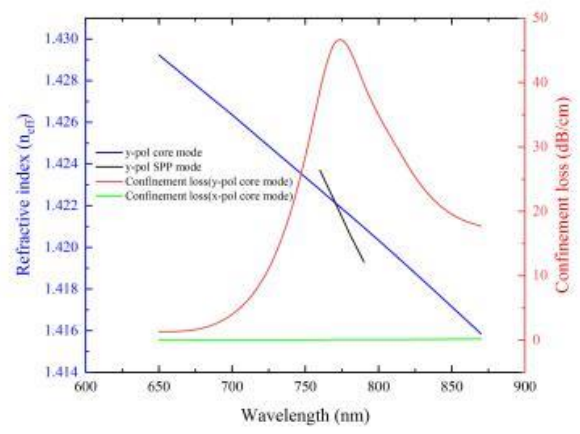

(a)

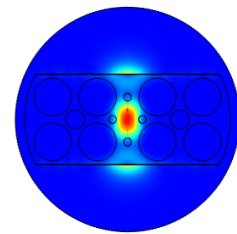

(b)

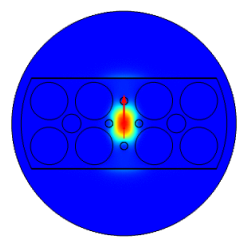

(d)

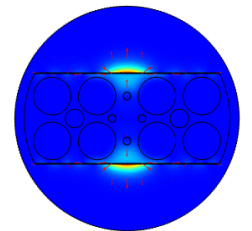

(c)

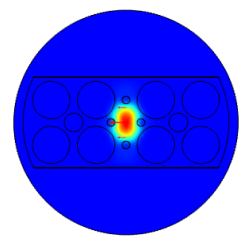

(e)

Fig. 2. (a)The dispersion relationship of core-guided mode, SPP mode and confinement loss spectrum at RI=1.38. (b) y-polarized core mode at $\lambda=774 \mathrm{~nm}$ (phase matching point); (c) y-polarized SPP mode at $\lambda=760 \mathrm{~nm}$; (d) x-polarized core mode at $\lambda=760$ $\mathrm{nm}$; (e) y-polarized core mode at $\lambda=750 \mathrm{~nm}$.

The coupling characteristics of the two-side polishing mold PCF-SPR sensor are the same as those of the common PCF-SPR sensor. Fig. 2 (a) shows the confinement loss spectra of the core modes in the $\mathrm{x}$-polarization (x-pol), y-polarization (y-pol) and the dispersion relationship between the core model in the y-direction and the SPP mode when the refractive index of the analyte is 1.38. When the real part of the effective refractive index of the core mode is equal to that of the SPP mode, the two modes will be coupled to achieve the maximum energy transmission between the two modes, and the loss value will be the highest. The electric field diagram is shown in Fig. 2 (b). Fig. 2 (c) to (e) are the electric field diagrams of SPP mode, y-pol core mode, and x-pol core mode at the wavelength of $760 \mathrm{~nm}$ respectively. It can be seen that the loss value of the core mode of x-pol is extremely low. Therefore, subsequent studies are based on the core mode of y-pol.

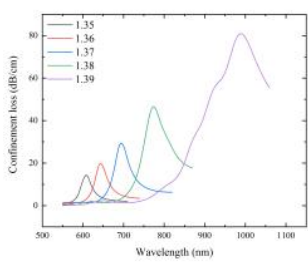

(a)

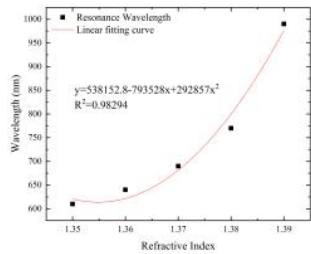

(c)

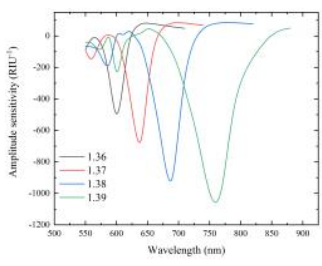

(b)

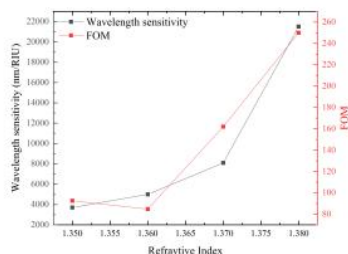

(d)

Fig. 3. Sensor performance. (a) Confinement loss spectra from 1.35 to 1.39. (b) Amplitude sensitivity spectra from 1.36 to 1.39 .

(c) linear curve fitting of the resonant wavelength; (d) Wavelength sensitivity and FOM from 1.35 to 1.38 .

The double-polishing PCF-SPR sensor presented in this paper has good sensing performance. As can be seen from Fig. 3 (a), with the increase of the refractive index of the solution to be measured, the 
resonance wavelength is red-shifted and the limiting loss increases. The reason for this phenomenon is that when the RI refractive index of the liquid to be tested increases, the evanescent field becomes stronger, leading to stronger coupling. At the same time, the effective refractive index of SPP mode increases while the core model remains unchanged. Fig.3 (b) shows the amplitude sensitivity of the sensor, which is the largest when the liquid to be tested RI is 1.39 and is $-1057.1756 \mathrm{RIU}^{-1}$. Fig. 3 (c) is the linear polynomial fitting of resonance wavelength, $\mathrm{R}^{2}=0.98265$, which proves that the sensor has a good continuous working capability. Fig. 3 (d) shows wavelength forest sensitivity and FOM, with the maximum wavelength sensitivity of $21500 \mathrm{~nm} / \mathrm{RIU}$ and the maximum FOM of 250.

Table 1 Performance comparison of the sensor with recently reported sensors

\begin{tabular}{|c|c|c|c|c|c|c|}
\hline Fiber Structure & $\begin{array}{l}\text { Refractive Index } \\
\text { (RIU) }\end{array}$ & $\begin{array}{l}\text { Amplitude } \\
\text { Sensitivity } \\
\left(\mathrm{RIU}^{-1}\right)\end{array}$ & $\begin{array}{l}\text { Wavelength } \\
\text { Sensitivity } \\
\text { (nm/RIU) }\end{array}$ & $\begin{array}{l}\text { Wavelength } \\
\text { Resolution } \\
\text { (RIU) }\end{array}$ & FOM & $\begin{array}{l}\text { Published Year } \\
\text { and Ref }\end{array}$ \\
\hline Double core PCF & $1.45-1.48$ & -700 & 8000 & $1.25 \times 10^{-5}$ & 138 & $2019[29]$ \\
\hline Double sided polishing & $1.30-1.342$ & N/A & 8000 & $1.3 \times 10^{-5}$ & N/A & $2019[30]$ \\
\hline \multicolumn{7}{|l|}{ PCF } \\
\hline The pores are filled with & $1.454-$ & N/A & 8776.8 & N/A & 168.16 & $2020[31]$ \\
\hline metal PCF & 1.478 & & & & & \\
\hline annular microfluidic channel & $1.33-1.36$ & -633.40001 & 10600 & $9.43 \times 10^{-5}$ & 303 & $2020[32]$ \\
\hline \multicolumn{7}{|l|}{ PCF } \\
\hline Side-polished & $1.35-1.39$ & N/A & 12500 & $8.0 \times 10^{-6}$ & N/A & $2021[33]$ \\
\hline \multicolumn{7}{|l|}{ PCF } \\
\hline Curved polished PCF & $1.36-1.402$ & -757 & 7142.86 & $3.2 \times 10^{-5}$ & N/A & $2021[15]$ \\
\hline Our work & $1.35-1.39$ & -1057.1756 & 21500 & $4.65 \times 10^{-6}$ & 250 & \\
\hline
\end{tabular}

Table 1 shows the performance comparison between the proposed sensor and the sensor published in the last three years. It can be seen from Table 1 that the dual-polished PCF-SPR biosensor proposed in this paper has a better performance compared with other PCF-SPR biosensors, and the same performance is also due to the previously published dual-polished PCF-SPR biosensor.

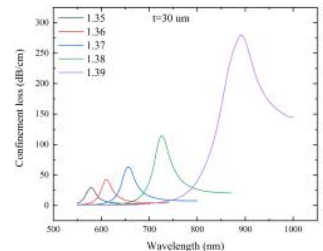

(a)

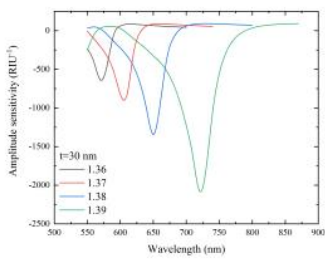

(d)

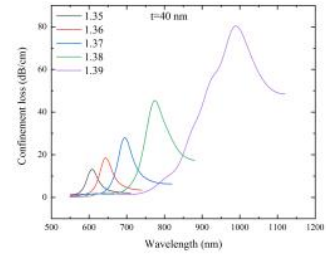

(b)

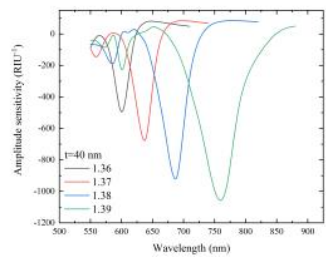

(e)

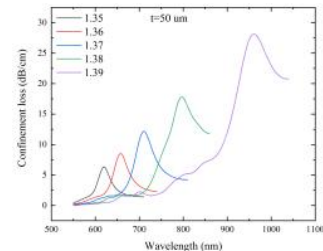

(c)

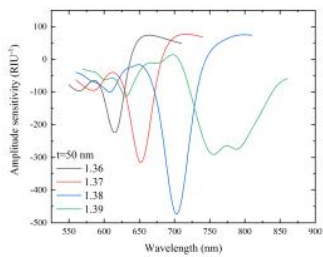

(f)

Fig. 4. (a)-(c) Confinement loss spectra with varying silver thicknesses and (d)-(f) Amplitude sensitivity spectra with varying silver thicknesses.

The influence of metal film thickness on sensing performance is great. Fig.4 (a), (b), and (c) 
respectively show the confinement loss spectra of RI from 1.35 to 1.39 when the thickness of the gold layer is $30 \mathrm{~nm}, 40 \mathrm{~nm}$, and $50 \mathrm{~nm}$. Fig. 4 (d), (e), and (f) is the amplitude sensitivity of the gold layer with $30 \mathrm{~nm}, 40 \mathrm{~nm}$, and $50 \mathrm{~nm}$ thickness. It can be seen that with the increase of metal thickness, the confinement loss of the same refractive index decreases, and the amplitude of the amplitude sensitivity decreases. This is because the transfer of light from the core to the analyte is reduced by the thicker gold layer. However, the actual manufacturing and use will cause losses to the gold layer, so we choose the gold layer thickness of $40 \mathrm{~nm}$.

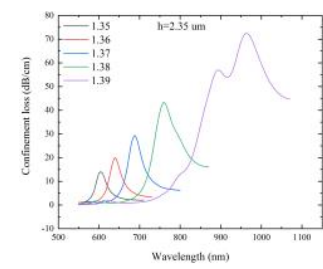

(a)

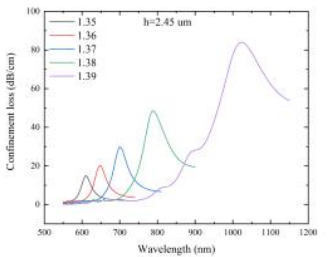

(c)

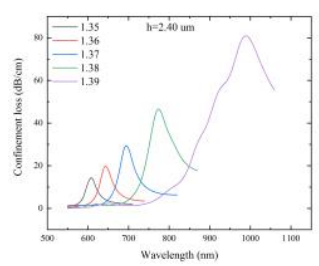

(b)

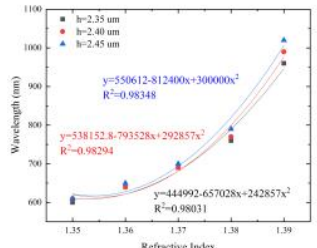

(d)

Fig. 5. Confinement loss spectra with varying polishing depth $h$. (a) $h=2.35 \mathrm{um}$; (b) $\mathrm{h}=2.40 \mathrm{um}$; (c) h=2.45 um. (d) Polynomial linear fitting.

The depth of the polishing mode has a great influence on the dual-polishing photonic crystal fiber. As can be seen from Fig. 5 (a) to (c), with the decrease of polishing depth(h increased), the confinement loss of the core mode increases, and the resonance wavelength redshift, but the wavelength sensitivity decreases. This is because the distance between the core mode and the SPP mode decreases with the decrease of the polishing depth, and the coupling phenomenon is more likely to occur. Fig. 5 (d) is a polynomial fitting curve, $\mathrm{R}^{2}$ decreases with the decrease of polishing depth. Combined sensitivity, confinement loss, and linear relationship, h was chosen as $2.40 \mathrm{um}$. This also proves that double-sided polishing PCF can solve the problem of high loss caused by the increase of polishing depth of D-shape PCF.

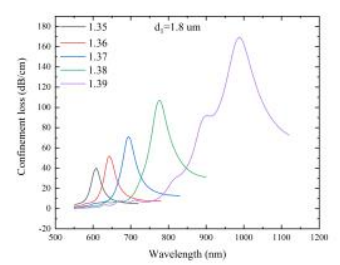

(a)

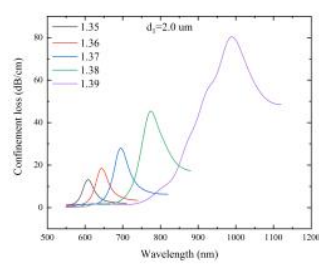

(b) 


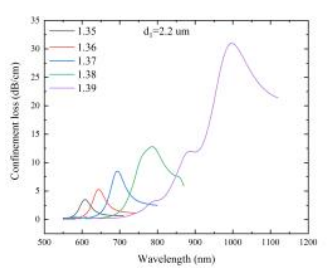

(c)

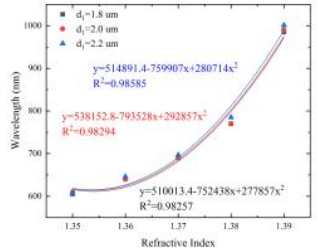

(d)

Fig. 6. Confinement loss spectra with varying $d_{1}$. (a) $d_{1}=1.8 u m$; (b) $d_{1}=2.0 u m ;$ (c) $d_{1}=2.2$ um; (d) Polynomial linear fitting.

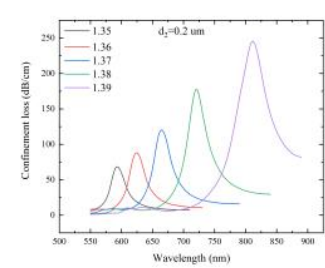

(a)

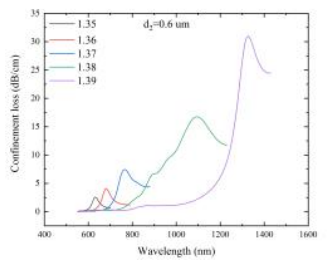

(c)

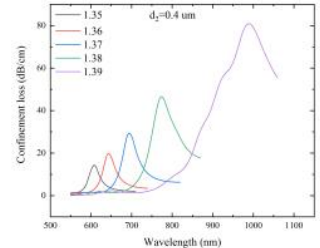

(b)

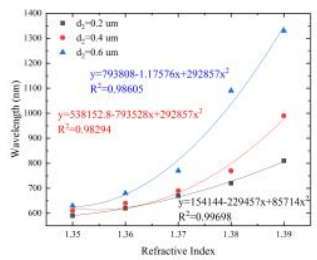

(d)

Fig. 7. Confinement loss spectra with varying $d_{2}$. (a) $d_{2}=0.2$ um; (b) $d_{2}=0.4$ um; (c) $d_{2}=0.6$ um; (d) Polynomial linear fitting.

Then we analyze the influence of air holes on the sensing performance. Fig. 6 to Fig. 8 respectively analyze the influence of $d_{1}, d_{2}$, and $d_{3}$ of different sizes on the sensor performance. It can be seen from Fig. 6 and Fig. 7 that $d_{1}$ and $d_{2}$ have similar effects on the sensor. With the increase of diameter, the resonance wavelength is redshifted, the confinement loss is reduced and the sensitivity is increased. As the diameter of the two air holes increases, the coupling channel width between the core mode and the SPP mode decreases, making the coupling phenomenon more difficult to occur. Since the air hole $d_{2}$ is in the middle of the coupling channel, it has a greater impact on performance. Fig. 6 (d) and Fig. 7 (d) are polynomial linear fitting curves of different $d_{1}$ and $d_{2}$ respectively. The change of $R^{2}$ after the same air hole size can also prove that the $\mathrm{d}_{2}$ size on the coupling channel has a greater impact on the sensing performance. Combined sensitivity and confinement loss, $d_{1}$ and $d_{2}$ are set to 2.0 um and 0.4 um, respectively.

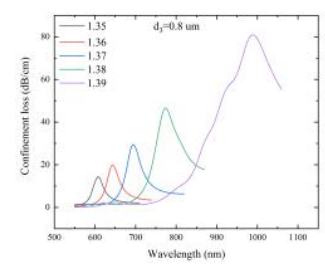

(a)

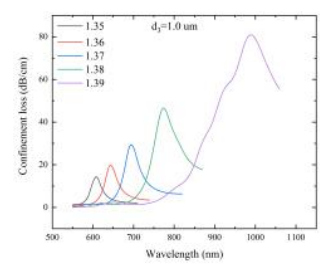

(b) 


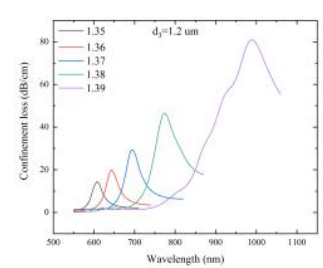

(c)

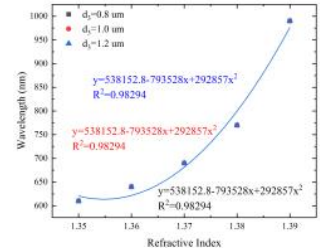

(d)

Fig. 8. Confinement loss spectra with varying $\mathrm{d}_{3}$. (a) $\mathrm{d}_{3}=0.8 \mathrm{um}$; (b) $\mathrm{d}_{3}=1.0 \mathrm{um}$; (c) $\mathrm{d}_{3}=1.2 \mathrm{um}$; (d)Polynomial linear fitting.

From Fig. 8 (a) to (c), it can be seen that the diameter of air hole $d_{3}$ does not influence sensor performance. That's because it has no contact-coupled channel. However, air hole $\mathrm{d}_{3}$ can limit the location of the core mode and increase the fault tolerance of the fabrication.

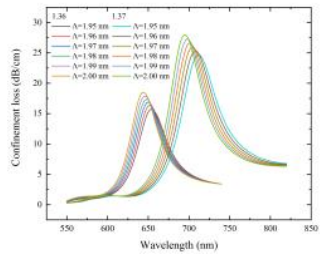

(a)

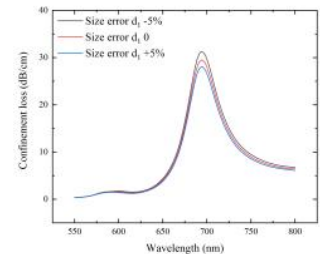

(b)

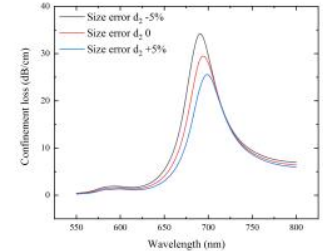

(c)

Fig. 9. (a) Confinement loss spectra with varying pitch size; (b) Confinement loss spectra with varying size error.

Fig. 9 (a) shows the confinement loss spectra with varying pitch sizes. It can be seen that with the increase of the interval, the resonance wavelength shifts blue, the loss increases, and the sensitivity changes little. This is due to the increase of spacing, the coupling channel between the core mode and the SPP mode becomes wider, and the coupling phenomenon is more likely to occur. Generally, the manufacturing error of photonic crystal fiber is within 5\%. Fig. 9 (b) and (c) show the loss spectrum with plus or minus $5 \%$ error (Fiber air hole size) at RI of 1.37 . It can be seen that when the size error exists in the fabrication of photonic crystal fiber, the influence on the resonance wavelength is small, but the influence on the confinement loss is large.

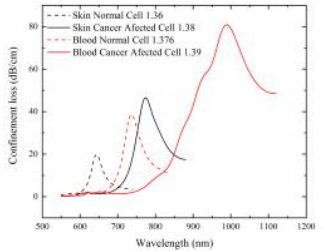

(a)

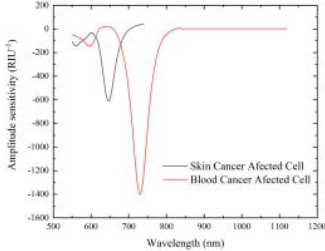

(b)

Fig.10. (a) Confinement loss spectra with a skin cell and blood cell; (b)Amplitude sensitivity.

The biosensor proposed in this paper can be applied in the field of cancer detection. Fig. 10 shows the sensing performance of this sensor for specific applications in the cancer detection field. According to the existing literature, the refractive index of normal skin cells is 1.36 , after canceration, the refractive index of cells was 1.38. The refractive index of normal blood cells is 1.376 , and after canceration, the refractive index of cells was 1.39 [16]. The sensor can be applied to detect these two cancerous phenomena with a sensitivity of $6500 \mathrm{~nm} / \mathrm{RIU}$ and $17875 \mathrm{~nm} / \mathrm{RIU}$ and amplitude sensitivity of 642.60477 $\mathrm{RIU}^{-1}$ and $-1478.69277 \mathrm{RIU}^{-1}$ respectively. It has very superior performance. 


\section{Conclusions}

In conclusion, a biosensor with double-sided polishing film PCF-SPR coated with the nanoscale gold film is proposed, and the dispersion relationship and modal loss analysis are carried out by using the finite element method. The performance of the biosensor was analyzed by using wavelength and amplitude interrogation methods. The maximum wavelength sensitivity is $21500 \mathrm{~nm} / \mathrm{RIU}$, the average wavelength sensitivity is $9550 \mathrm{~nm} / \mathrm{RIU}$, the amplitude sensitivity is $-1057.1756 \mathrm{RIU}^{-1}, \mathrm{R}^{2}$ is 0.98265 , FOM is 250 and the maximum resolution is $4.68 \times 10^{-8}$, which can realize the monitoring between RI 1.35-1.39. It has a wavelength sensitivity of $17875 \mathrm{~nm} / \mathrm{RIU}$ and the amplitude sensitivity of -1478.69277 $\mathrm{RIU}^{-1}$ in the detection of the blood cancer cell. The sensor has excellent performance and can be applied to single cell detection of cancer.

\section{Declarations}

Funding. The National Natural Science Foundation of China [grant number 52102164]; the Equipment pre-research field fund project [grant number61404140521] and supported by the Fundamental Research Funds for the Central Universities [grant number 021314380095] and Digit Fujian Internet-of-Things Laboratory of Environmental Monitoring Research Fund (Fujian Normal University) (NO. 202002).

Conflicts of interest. The authors declare no conflicts of interest.

Availability of data and material. The datasets generated during and/or analysed during the current study are available from the corresponding author on reasonable request.

Code availability. Not applicable.

Ethics approval. Not applicable.

Authors' contributions. All authors contributed to the study conception and design. Material preparation, data collection and analysis were performed by Zhiwen Zhang. The first draft of the manuscript was written by Zhiwen Zhang and all authors commented on previous versions of the manuscript. All authors read and approved the final manuscript.

Consent to participate. Not applicable.

Consent for publication. Not applicable.

\section{reference}

[1] Singh, P (2016) SPR biosensors: Historical perspectives and current challenges. Sens. Actuators B Chem $229,110-130$.

[2] Sharma, A.K., Jha, R. (2007) Gupta, B.D, Fiber-optic sensors based on surface plasmon resonance: A comprehensive review. IEEE Sens. J 7 1118-1129.

[3] Klantsataya E, Jia P, Ebendorff-Heidepriem H, Monro T.M, Francois A (2016) Plasmonic fiber-optic refractometric sensors: From conventional architectures to recent design trends. Sensors, 17, 12.

[4] E. Kretschmann, H. Raether (1968) Notizen: radiative decay of nonradiative surface plasmons excited by light. Z. Naturforsch 23 (12), 2135-2136.

[5] E. Haque, H.M. Anwar, Y. Namihira, F (2019) Ahmed, Microchannel-based plasmonic refractive Index sensor for low refractive Index detection. Appl. Optic 58 (6), 1547-1554.

[6] Klantsataya E, François A, Ebendorff-Heidepriem H, Hoffman P, Monro T (2015) Surface plasmon scattering in exposed core optical fiber for enhanced resolution refractive index sensing. Sensors 15 25090-25102. 
[7] Rifat A.A, Mahdiraji G.A, Chow D.M, Shee Y.G, Ahmed Y.G, Adikan F.R.M (2015) Photonic crystal fiber-based surface plasmon resonance sensor with selective analyte channels and graphene-silver deposited core, Sensors 15 , 11499-11510.

[8] A.A. Rifat, R. Ahmed, A.K. Yetisen, H. Butt, A. Sabouri, G.A. Mahdiraji, S.H. Yun, F.M. Adikan (2017) Photonic crystal fiber-based plasmonic sensors. Sens. Actuators B Chem 243 May 1, 311-325. [9] S. Fatema, R. Absar, M.I. Reja, J. Akhtar (2017) Effect of core infiltration in the birefringence of Photonic Crystal Fiber. IEEE International Conference on Telecommunications and Photonics (ICTP) December 26, IEEE pp. 1-5.

[10] S. Fatema, R. Absar, M.I. Reja, J. Akhtar, Effect of soft glass rod infiltration in the core of photonic crystal fiber (2020) J. Opt. Commun 41 (4) April 28, 371-383.

[11] Hassani A, Skorobogatiy, M (2007) Design criteria for microstructured-optical fiber-based surface plasmon resonance sensors. J. Opt. Soc. Am. B, 24 , 1423-1429.

[12] Peng Y, Hou J, Huang Z, Lu Q (2012) Temperature sensor based on surface plasmon resonance within selectively coated photonic crystal fiber Appl. Opt 51, 6361-6367.

[13] Sharma P, Sharan P, Deshmukh P (2015) A photonic crystal sensor for analysis and detection of cancer cells. International Conference on Pervasive Computing (ICPC). IEEE pp. 1-5.

[14] Lu, Junjie, et al. (2018) D-shaped photonic crystal fiber plasmonic refractive index sensor based on gold grating. Applied optics 57.19 5268-5272.

[15] Ahmet Yasli, (2021) Cancer Detection with Surface Plasmon Resonance-Based Photonic Crystal Fiber Biosensor. Plasmonics 15 March.

[16] Tsai C, Huang S, (2012) Water distribution in cancer and normal cells.

[17] Haque E, Hossain MA, Ahmed F, Namihira Y (2018) Surface plasmon resonance sensor based on modified D-shaped photonic crystal fiber for a wider range of refractive index detection. IEEE Sens J 18 (20):8287-8293.

[18] L. Armelao, D. Barreca, G. Bottaro, G. Bruno, A. Gasparotto, M. Losurdo, E. Tondello (2005) RFsputtering of gold on silica surfaces: evolution from clusters to continuous films. Mater. Sci. Eng., C 25 599-603.

[19] G.V. Naik, V.M. Shalaev, A. Boltasseva (2013) Alternative Plasmonic Materials: Beyond Gold and Silver. Adv Mater 25 3264-3294.

[20] R.Z. Li Pei, Tigang Ning, Chunhui Qi, Jing Li (2010) Key technologies for side-grinding optical fiber with long-length and high-precision and their applications. Infrared Laser Eng 1022.

[21] M.E. Gorachand Ghosh, Member, IEEE, Takashi Iwasalu (1994) Temperature-dependent Sellmeier coefficients and chromatic dispersions for some optical fiber glass. J. Lightwave Technol 12 1338-1342.

[22] Gururaj V. Naik, Jeremy L. Schroeder, Xingjie Ni, Alexander V. Kildishev (2012) Timothy D. Sands, Alexandra Boltasseva, Titanium nitride as a plasmonic material for visible and near-infrared wavelengths. Opt Mater Exp 2 (4) 478-489.

[23] Ahmmed A. Rifat, Md Rabiul Hasan, Rajib Ahmed, Haider Butt, Photonic crystal fiber-based plasmonic biosensor with external sensing approach. J Nanophoton 12 (1) (2017) 012503.

[24] C. Liu, L. Yang, X. Lu, Q. Liu, F. Wang, J. Lv, T. Sun, H. Mu, and P. K. J. O. E. Chu, Mid-infrared surface plasmon resonance sensor based on photonic crystal fibers. Opt. Express 25 (2017) 14227-14237. [25] Dash Jitendra Narayan, Rajan Jha (2014) SPR biosensor based on polymer PCF coated with conducting metal oxide. IEEE Photon Technol Lett 26 (6), 595-598. 
[36] E. Haque, M.A. Hossain, F. Ahmed, Y. Namihira (2018) Surface plasmon resonance sensor based on modified D-shaped photonic crystal fiber for a wider range of refractive index detection. IEEE Sensor. J. 1-1.

[27] F. Haider, R. A. Aoni, R. Ahmed, A. E. Miroshnichenko (2018), Highly amplitude-sensitive photonic-crystal-fiber-based plasmonic sensor. J. Opt. Soc. Am. B 35 2816-2821.

[28] Ahmed H. El-Saeed, Ahmed E. Khalil, Mohamed Farhat O, Hameed Mohammad Y. Azab, S. S. A. Obayya (2019) Highly sensitive SPR PCF biosensors based on Ag/TiN and Ag/ ZrN configurations. Optical and Quantum Electronics 51:56.

[29] Md. Nazmus Sakib, Mb. Biplob Hossain, Kusay Faisal Al-tabatabaie, Ibrahim Mustafa Mehedi, Md. Tanvir Hasan, Md. Amzad Hossain, I.S.Amiri (2019) High-Performance Dual Core D-Shape PCF-SPR Sensor Modeling Employing Gold Coat. Results in Physics..

[30] Shun Wang, Shuguang Li (2019) Surface plasmon resonance sensor based on symmetrical sidepolished dual_core photonic crystal fiber. Optical Fiber Technology 51, 96-100.

[31] Qiang Liu, Zhuang Ma, Qiang Wub, Weilin Wang (2020) The biochemical sensor based on liquidcore photonic crystal fiber filled with gold, silver, and aluminum. Optics and Laser Technology 130 106363.

[32] Hairui Fang, Changing Wei, Dong Wang, Long Yuan, Shengxi Jiao, Zhiyu Bao, and Hanrui Yang (2020) Research on photonic crystal fiber based on a surface plasmon resonance sensor with segmented silver-titanium dioxide film. Journal of the Optical Society of America B Vol. 37, No. 3.

[33] Yujun Wang, Shuguang Li, Mingyue Wang, Pengtao Yu (2021) Refractive index sensing and filtering characteristics of side-polished and gold-coated photonic crystal fiber with an offset core. Optics \& Laser Technology 136, 106759. 